\title{
Modeling the Force-Velocity Relationship in Arm Movement
}

\author{
Ahti Rahikainen, Janne Avela, Mikko Virmavirta \\ Department of Biology of Physical Activity, University of Jyväskylä, Neuromuscular Research Center, Jyväskylä, Finland \\ Email: ahrahik.zz@kolumbus.fi
}

Received February 1, 2012; revised March 2, 2012; accepted March 17, 2012

\begin{abstract}
Modeling the force-velocity dependence of a muscle-tendon unit has been one of the most interesting objectives in the field of muscle mechanics. The so-called Hill's equation [1,2] is widely used to describe the force-velocity relationship of muscle fibers. Hill's equation was based on the laboratory measurements of muscle fibers and its application to the practical measurements in muscle mechanics has been problematic. Therefore, the purpose of this study was to develop a new explicit calculation method to determine the force-velocity relationship, and test its function in experimental measurements. The model was based on the motion analysis of arm movements. Experiments on forearm rotations and whole arm rotations were performed downwards and upwards at maximum velocity. According to the present theory the movement proceeds as follows: start of motion, movement proceeds at constant maximum rotational moment (Hypothesis 1), movement proceeds at constant maximum power (Hypothesis 2), and stopping of motion. Theoretically derived equation, in which the motion proceeds at constant maximum power, fitted well the experimentally measured results. The constant maximum rotational moment hypothesis did not seem to fit the measured results and therefore a new equation which would better fit the measured results is needed for this hypothesis.
\end{abstract}

Keywords: Muscle Mechanics; Muscle Power; Force-Velocity Relationship; Arm Movement

\section{Introduction}

Modeling the force-velocity relationship of muscle-tendon unit involves many different factors. In muscle mechanics force-velocity relationship of skeletal muscle is often presented by so-called Hill's equation $(F+a)(v+b)$ $=b\left(F_{0}+a\right)$, where $F$ is the maximum force within muscle contraction, $a$ and $b$ are constants, $F_{0}$ the isometric force of muscle or the constant maximum force generated by muscle with zero velocity and $v$ is velocity, (Figure 1) [1,2]. This equation was based on the laboratory measurements in which force $(F)$ of the activated muscle lifted different loads $(F=m g)$ and speed of the load ( $v$ ) was then measured. In Hill's equation $F$ is force, $a$ is constants force, $v$ is velocity, $b$ is constant velocity and $F_{0}$ is constant force. In the equation the vectors of forces and velocities have the same direction and therefore Hill's equation can be presented in a scalar form. The left side of Hill's equation is the product of force and velocity and that is power. As the right side of the equation is constant it can be seen that Hill's equation is a constant power model. Hill's force-velocity relationship is one of the most essential equations of muscle mechanics and it has often been principle object in biomechanical studies for about 50 years, e.g. [3-6]. Force measured from skeletal muscle during maximum tension depends on several internal and external factors. Internal factors are e.g. anatomical structure of muscle (cross sectional area, pennation etc.), fiber type distribution (fast and slow twitch muscle fibers have different force-velocity equations), condition of the muscle (fatigue, training) and muscle length. External factors are e.g. contraction type (isometric, concentric and eccentric) and contraction velocity (rate of change of muscle length). Good reviews of the above mentioned factors have been presented, e.g. $[4,7,8]$. Force $(F)$ creates a moment about the joint which is moment arm multiplied by force $(M=r \times F)$. Length of muscle's moment arm depends on joint angle and it changes as the rotation movement proceeds about the joint axis. The combined effect of the forces of several different muscles produces the rotation movement about the joint axis.

Due to all the above mentioned factors it is difficult to determine the force production $[9,10]$, and also to determine the torque about the joint. The purpose of this study was to develop a new explicit calculation method to determine the force-velocity relationship and test its function in experimental measurements. This method is based on the assumption that in muscle mechanics there exists a constant maximum power which the muscle is able to 


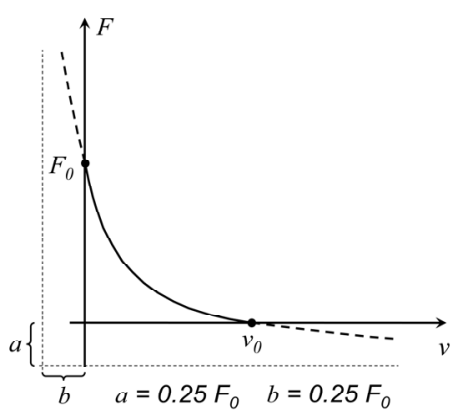

Figure 1. Hill's equation $(F+a)(v+b)=b\left(F_{0}+a\right)$ where $F_{0}$ is so-called isometric force or force with zero velocity, $v_{0}$ is the highest possible velocity, $a$ and $b$ are constant force and constant velocity. In rotational movement torque $M$ corresponds to force $F$ and angular velocity $\varphi$ corresponds to velocity $v$.

generate within a certain range of velocity. The principle of constant maximum power is the same as in Hill's equation except that the constant maximum power in the present study is a characteristic of whole muscle group instead of separate muscle fibers as in the Hill's equation. This study continues the development of the earlier findings [11-13].

\section{Methods}

The experiments in the present study consisted of three different maximum velocity arm movements: 1) forearm rotation downwards, 2) whole arm rotation downwards and 3) upwards. The selection of these movements was based on the earlier findings of Rahikainen and Luhtanen [11] where so called "constant power theory" seemed to work at the last phase of the arm push in shot put. In order to study this finding more extensively it was reasonable to choose a simple procedure as represented by arm rotations in the present study. The photographs of arm movements in this study were generated by a special motion camera system $[14,15]$ which represents the movement as a series of object images. The paths of the mark lights attached to the moving object can be seen as broken light-lines. The principle of the method is to photograph the moving object through a rotating disc which consists of one transparent opening and nine filter openings serving as the shutter apertures. As the exposure disc rotates in front of the camera lens (film camera $\mathrm{Ca}$ non T70) and the camera aperture is open, the disc serves as the shutter. This way several overlapping exposures are generated on the same frame. The transparent opening generates images of the moving object, and the filter openings generate the light-lines indicating the paths of mark lights attached to the moving object (Figure 2). In this study the speed of rotation of the exposure disc was 300 rotations per minute, exposing five (300/60) object images per second and giving the time interval of $20 \mathrm{~ms}$

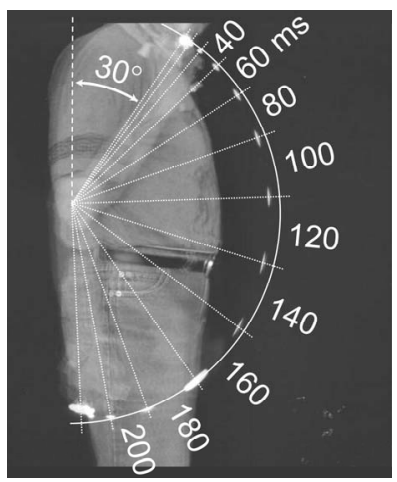

Figure 2. Forearm rotation downwards with maximum force. Angle of rotation $\varphi$ and its corresponding time $T(\mathrm{~ms})$ are presented on the subject image.

for nine light-lines between consecutive object images (for more detail, see [14,15]). Figure 2 represents a forearm rotation downwards. As seen in the figure the radius of the rotation circle is not exactly the same as the radius of forearm rotation. This is because of a slight motion of the elbow joint. Actually the radius of forearm rotation is slightly larger than the radius of circle on the figure and it can be measured from the forearm image before the start of the rotation movement. Angular velocity measurements are calculated with the formula

$$
\dot{\phi}=\Delta S / R \Delta T
$$

in which the length of forearm is the radius of rotation $R$ and the distance measured between two successive measured points on the path of light-lines is the distance increment $\Delta S$.

\subsection{Measurement of Rotation Arc}

For convenience the arc $\Delta S_{1}$ was measured as a straight line $\Delta S_{2}$ (Figure 3) and the error between these two values was estimated. The arc $\Delta S_{1}$ can be calculated from the straight line $\Delta S_{2}$ from the formula:

$$
\Delta S_{1}=R \arccos \left(1-\frac{1}{2}\left(\frac{\Delta S_{2}}{R}\right)^{2}\right)
$$

Formula derivation from the right-angled triangle in Figure 3.

$$
\begin{gathered}
\Delta S_{2}^{2}=R^{2} \sin ^{2} \Delta \varphi+R^{2}(1-\cos \Delta \varphi)^{2} \\
\left(\frac{\Delta S_{2}}{R}\right)^{2}=\sin ^{2} \Delta \varphi+\cos ^{2} \Delta \varphi+1-2 \cos \Delta \varphi \\
\sin ^{2} \Delta \varphi+\cos ^{2} \Delta \varphi=1 \\
\left(\frac{\Delta S_{2}}{R}\right)^{2}=2-2 \cos \Delta \varphi
\end{gathered}
$$




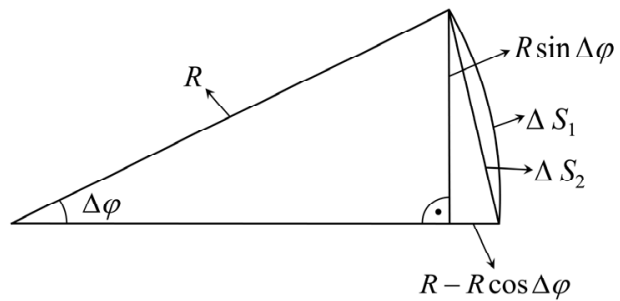

Figure 3. Measurement of the rotation arc. $\varphi=$ angle of rotation, $R=$ length of forearm, arc $\Delta S_{1}=$ distance the mark light travels during the time interval $\Delta t$ and $\Delta S_{2}=$ the arc $\Delta S 1$ measured as a straight line.

$$
\begin{gathered}
\cos \Delta \varphi=1-\frac{1}{2}\left(\frac{\Delta S_{2}}{R}\right)^{2} \\
\Delta S_{1}=R \arccos \left(1-\frac{1}{2}\left(\frac{\Delta S_{2}}{R}\right)^{2}\right)
\end{gathered}
$$

The maximum value measured from Figure 2 (corresponding time $140 \mathrm{~ms}$ ) is $\Delta S / R=\Delta S_{2} / R=0.356$. Substituting this value in the formula above (7) the arc of rotation is obtained as ratio form $\Delta S_{1} / R=0.358$. It can be seen that $\Delta S_{2}$ fits with adequate accuracy to the distance $\Delta S_{1}$.

\subsection{Progress of Research}

The present study continues the earlier study [11] and it is a new round in the diagram of Figure 4 presenting the progress of research (testing the hypotheses): 1) Equation of arm movement was derived and test predictions were made. 2) Experiments were performed in arm rotations. 3) Equation of arm rotation was fitted to the experimental results and their compatibility was observed. 4) If the present equation of motion did not fit at all the measuring results, the hypothesis would be disproved. If the present equation of motion fitted the measuring results in some definite accuracy, the hypothesis would receive confirmation. 5) In the future, by making additional experiment (a new round in the diagram) the hypothesis will receive more confirmation.

\subsection{Arm Rotation}

Because the muscle system is able to transfer only a certain quantity of chemical energy during the time of contraction, it is obvious that arm rotation must have maximum power that cannot be exceeded. It can also be assumed that the maximum power acts within a certain range of velocity and it is a constant maximum power. At the beginning of the movement angular velocity is naturally zero and it takes some time to generate force. After the start of the movement it is possible that a maximum muscle force takes action and within rotational motion maximum rotational moment acts as well. The constant

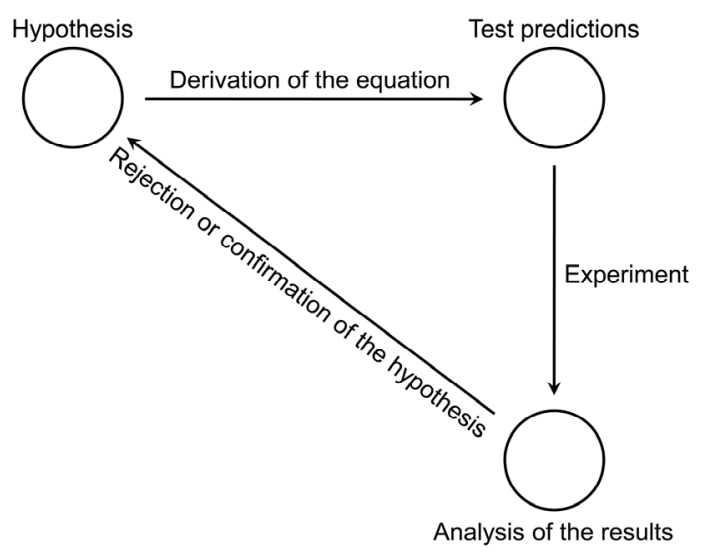

Figure 4. Diagram of the progress of testing the hypotheses of arm rotations.

maximum power acts within a certain range of velocity which cannot be at the beginning of the rotational movement because power is the product of moment and angular velocity. Therefore, a constant power "theory" is possible only when the velocity is high enough. As the velocity increases the motion reaches the point where the maximum power takes action and acting rotational moment is less than the maximum moment. This way power remains constant as the angular velocity increases and moment decreases.

\subsection{Research Hypotheses}

According to the present theory and above mentioned facts the movement proceeds as follows: 1) start of motion, 2) movement proceeds at constant maximum rotational moment during the first part of the movement [Hypothesis 1], 3) movement proceeds at constant maximum muscular power during the second part of the movement [Hypothesis 2], 4) stopping of motion. In order to test the research hypotheses, the following experiments were conducted: forearm rotation downwards at maximum velocity (1), whole arm rotation downwards at maximum velocity (2), whole arm rotation upwards at maximum velocity (3). The maximum power hypothesis was tested so that the theoretical angular velocity-time values from Equation (15) were fitted into the measured angular velocity-time curves of arm rotations. It was assumed that if the measured angular velocity-time values matched the theoretical values within a certain velocity range then the Hypothesis 2 would be fulfilled. The maximum rotational moment hypothesis was tested by Equation (8).

\subsection{A Model of Arm Rotation}

It was assumed (hypothesis) that in muscle mechanics there exists the maximum power $(P)$ which the muscle is able to generate within a certain range of velocity. The 
Model of arm rotation was constructed according to Newton's II law which was applied to rotational motion where moment of inertia multiplied by angular acceleration equals rotational moment. Rotational moment equals moment generated by muscle force subtracted moment generated by inner friction of muscle. The effect of gravitational force is minor and it is added to the motion mechanics afterwards in Section 2.6. The model of arm rotation is the equation of motion:

$$
I \frac{\mathrm{d} \dot{\varphi}}{\mathrm{d} T}=\frac{P}{\dot{\varphi}}-C \dot{\varphi}
$$

where $I$ is moment of inertia in arm rotation, $\dot{\varphi}$ is angular velocity, $P$ is power generated by arm muscles, $T$ is time, $P / \dot{\varphi}$ is moment generated by muscle force, $C \dot{\varphi}$ is moment generated by inner friction of muscle and $C$ is constant coefficient of friction.

The mass distribution of the subject's arm sectors differed from the average values in subject mass tables. Therefore the mass distribution of the arm sectors were defined by sinking the arm sectors into water, and weighing the over flowed water. The masses of the arm sectors were calculated by means of water volume and arm sector density $(V * \rho)$. The length of subject's whole arm was $0.64 \mathrm{~m}$ and the arm sectors, hand, forearm 1 , forearm 2, upper arm 1, upper arm 2 were $0.128 \mathrm{~m}$ each. Arm sector densities were 1.16, 1.13, 1.07 for hand, forearm and upper arm, respectively [6]. Moment of inertia for the forearm rotation was $I=0.11 \mathrm{~kg} \cdot \mathrm{m}^{2}$ and for the whole arm rotation $I=0.52 \mathrm{~kg} \cdot \mathrm{m}^{2}$.

Hypothesis 1 implies that movement proceeds at a constant maximum rotational moment. In that case the moment generated by muscle force $P / \dot{\varphi}$ in Equation 8 is a constant maximum moment. Hypothesis 2 implies that movement proceeds at a constant maximum muscular power. In that case the power $P$ in Equation (8) is a constant maximum power. In order to determine the validity of Hypothesis 2, Equation (8) was solved for angular velocity-time function and this equation was employed for validity determination:

$$
\text { Equation of power } I \dot{\varphi} \frac{\mathrm{d} \dot{\varphi}}{\mathrm{d} T}=P-C \dot{\varphi}^{2}
$$

where $I \dot{\varphi} \frac{\mathrm{d} \dot{\varphi}}{\mathrm{d} T}$ is power in arm rotation, $P$ is power generated by arm muscles and $C \dot{\varphi}^{2}$ is power consumed by friction.

Solution

$$
\begin{gathered}
I \frac{\dot{\varphi}}{P-C \dot{\varphi}^{2}} \mathrm{~d} \dot{\varphi}=\mathrm{d} T \\
-\frac{I}{2 C} \int_{0}^{\dot{\varphi}}-2 C \dot{\varphi} \frac{1}{P-C \dot{\varphi}^{2}} \mathrm{~d} \dot{\varphi}=\int_{0}^{T} \mathrm{~d} T
\end{gathered}
$$

$$
\begin{gathered}
\ln \left(P-C \dot{\varphi}^{2}\right)-\ln (P)=-\frac{2 C}{I} T \\
\ln \left(\frac{P-C \dot{\varphi}^{2}}{P}\right)=-\frac{2 C}{I} T \\
1-\frac{C}{P} \dot{\varphi}^{2}=e^{-\frac{2 C}{I} T} \\
\dot{\varphi}=\sqrt{\frac{P}{C}\left(1-e^{-\frac{2 C}{I} T}\right)}
\end{gathered}
$$

\subsection{Effect of Gravitational Force on the Movement}

The moment which is induced by gravity $\sum r \times m g$ was omitted from the motion model. The power generated by this moment is $\left(\sum r \times m g\right) \dot{\varphi}$, where $m g$ is gravitational force of arm segments, $r$ is distance of the center of gravity of segments from the rotation axis and $\dot{\varphi}$ angular velocity of arm rotation. The theoretical angular velocity function, Equation (15), and the measured angular velocity function coincide within so narrow velocity range that the power induced by gravity can be calculated as a constant factor. In this case it is included in the power $P$ as follows: $P$ of rotation downwards $=$ power generated by muscular force + power generated by gravitational force and $P$ of rotation upwards: $P=$ power generated by muscular force-power generated by gravitational force.

\subsection{Finding the Matched Range of Measured and Theoretical Angular Velocity Functions}

There are two unknown variables in Equation (15), power $P$ and kinetic friction coefficient $C$. In order to determine these two unknown variables, two equations were required. These two equations were obtained from the hypothesis according to which the movement proceeds at constant maximum power within certain velocity range. By substituting two angular velocity-time value pairs from the measured angular velocity-time curve in Equation (15) the two required equations were obtained. The zero point of time (Figure 5) is at the intersection point of the time-axis and the broken-line curve and in order to find that some iteration was done. From these two equations $P$ and $C$ could be solved. Then the constant maximum power hypothesis was tested by comparing the calculated theoretical values from Equation (15) with the values of measured angular velocity-time curve.

\section{Results}

In Figure 5 the line (A-E) is connecting the experimental data points of Figure 2. Figure 6 shows the whole arm 


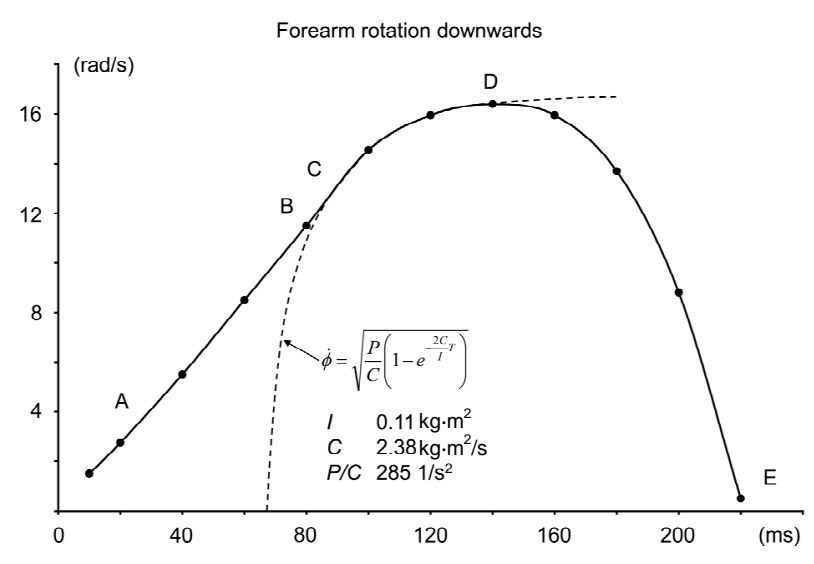

Figure 5. The measured angular velocities from forearm rotation downwards (points on the curve A-E) and the theoretical angular velocity values calculated from Equation 15 (broken line). The zero point of time for the theoretical angular velocity curve is at the intersection of the time-axis and the broken-line curve (the same time scaling is same for both curves).

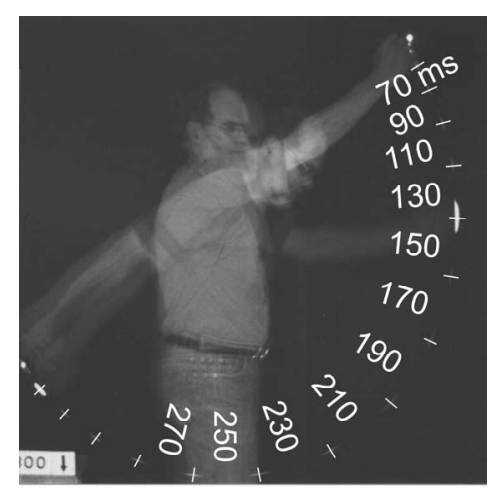

(a)

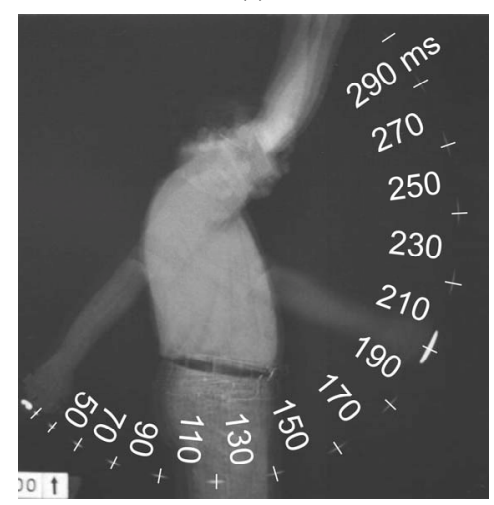

(b)

Figure 6. Whole arm rotation downwards (a) and upwards (b). Time of rotation is seen with the increment of $20 \mathrm{~ms}$.

rotations upwards and downwards. In Figures 5 and 7 the solid line is the curve fitting to the points representing the technique to filter small digitizing errors in traditional motion analysis. This way the complicated analysis of the series of the object images in the present study

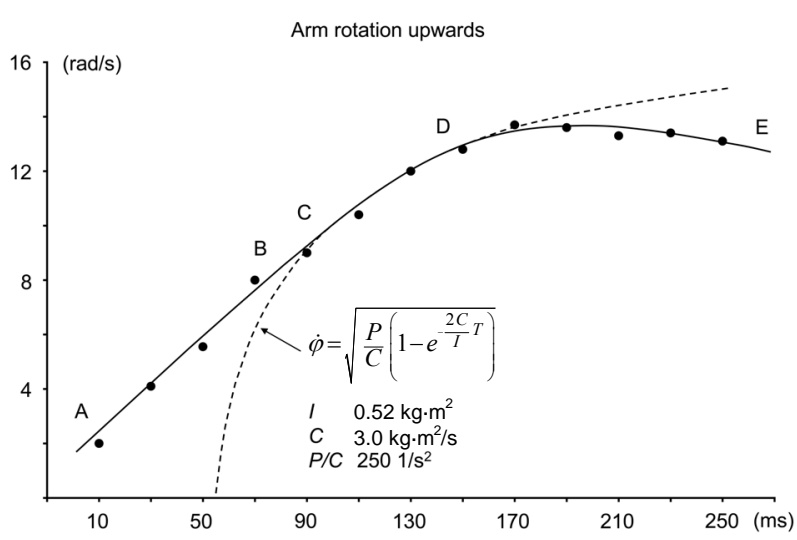

(a)

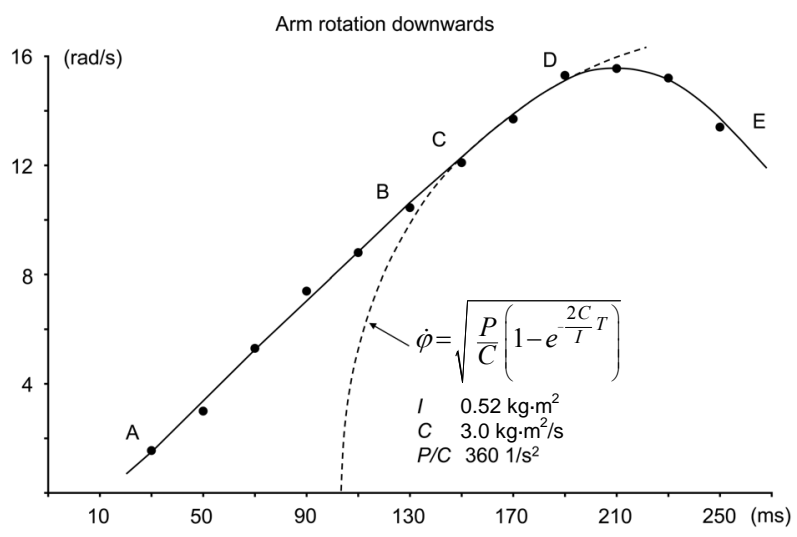

(b)

Figure 7. The measured angular velocities (points on the curve fitting A-E) from the whole arm rotation downwards (a) and upwards (b) and the theoretical angular velocity values calculated from Equation 15 (broken lines). The zero point of time for the theoretical angular velocity curve is at the intersection of the time-axis and the broken-line curve.

could be facilitated without losing a sufficient accuracy.

Hypothesis 1 states that the rotational movement proceeds at a constant maximum rotational moment within a certain range of velocity. This statement implies that rotational moment is about constant or $P / \dot{\varphi}$ is constant. By observing Figures 5 and 7 it can be seen that movement proceeds at constant acceleration or $\mathrm{d} \dot{\varphi} / \mathrm{d} T$ is constant approximately between the points $\mathrm{A}-\mathrm{B}$ on the velocity-time curve. The kinetic friction $C \dot{\varphi}$ is not constant. By substituting these terms in Equation (8)

$$
I \frac{\mathrm{d} \dot{\varphi}}{\mathrm{d} T}=\frac{P}{\dot{\varphi}}-C \dot{\varphi}
$$

it can be seen that the left side of the equation is constant and the right side of the equation is not constant. Therefore, we can conclude, that Hypothesis 1 is not fulfilled.

The measured values of the forearm rotation downwards are presented in Table 1. Angular velocities of the forearm rotation are shown in Figure $\mathbf{5}$ as points on the 
Table 1. Measured values of the forearm rotation downwards. Angular velocity is calculated with the Equation (1) and the angular acceleration according to Figure 7. A-B and $C-D$ represent the estimated phases where the movement proceeds at constant acceleration and constant power, respectively.

\begin{tabular}{cccccc}
\hline $\mathrm{T}(\mathrm{ms})$ & & $\Delta \varphi(\mathrm{rad})$ & $\Sigma \Delta \varphi(\mathrm{rad})$ & $\dot{\varphi}(\mathrm{rad} / \mathrm{s})$ & $\ddot{\varphi}\left(\mathrm{rad} / \mathrm{s}^{2}\right)$ \\
\hline 20 & & 0.055 & 0.06 & 2.75 & 114 \\
40 & $\mathrm{~A}$ & 0.110 & 0.17 & 5.50 & 155 \\
60 & & 0.170 & 0.35 & 8.52 & 155 \\
80 & $\mathrm{~B}$ & 0.231 & 0.59 & 11.54 & 155 \\
& $\mathrm{C}$ & & & & \\
100 & & 0.291 & 0.89 & 14.56 & 128 \\
120 & & 0.319 & 1.22 & 15.93 & 72.5 \\
140 & $\mathrm{D}$ & 0.329 & 1.56 & 16.48 & 12 \\
160 & & 0.319 & 1.89 & 15.93 & -56 \\
\hline
\end{tabular}

curve A-E. The theoretical angular velocity function with maximum power hypothesis (Equation 15) was fitted into the curve of the measured angular velocity-time values. Moment of inertia of forearm rotation was calculated $I=$ $0.11 \mathrm{~kg} \cdot \mathrm{m}^{2}$ (see 2.5 ). The values of friction coefficient $C$ and power and friction coefficient ratio $P / C$ were obtained within the curve fitting, $C=2.38 \mathrm{~kg} \cdot \mathrm{m} / \mathrm{s}^{2}$ and $P / C$ $=2851 / \mathrm{s}^{2}$. In Figure 5 the movement proceeds at a constant acceleration between the phases A and B $(\sim 40-80$ $\mathrm{ms})$ until the liquid friction begins to influence and acceleration decreases between B-C. According to the Hypothesis 2 the movement proceeds at a constant power between C-D which is followed by stopping of the movement (D-E). The theoretical angular velocity curve (broken line) coincides with the measured angular velocity curve within section C-D. Therefore, we conclude that Hypothesis 2 is fulfilled within this range of velocity.

Figure 7 represents the curves of the measured points of angular velocity-time values from the whole arm rotations downwards and upwards (Figure 6). The theoretical angular velocity functions with maximum power hypothesis (Equation (15)) were fitted into the measured point curves. Moment of inertia of forearm rotation was calculated $I=0.52 \mathrm{~kg} \cdot \mathrm{m}^{2}$ (see 2.5 ). The values of friction coefficient $C$ and power and friction coefficient ratio $P / C$ were obtained within the curve fitting, whole arm rotation downwards $C=3.0 \mathrm{~kg} \cdot \mathrm{m} / \mathrm{s}^{2}, P / C=3601 / \mathrm{s}^{2}$ and whole arm rotation upwards $C=3.0 \mathrm{~kg} \cdot \mathrm{m} / \mathrm{s}^{2}, P / C=250$ $1 / \mathrm{s}^{2}$. The movement follows the hypothesized movement pattern described in the forearm rotation above. The theoretical angular velocity curves (broken lines) coincide with the measured angular velocity curves in section C-D $(\sim 150-190 \mathrm{~ms}$ and $90-150 \mathrm{~ms}$ in downward and upward rotation, respectively, Figure 7).

\section{Validity and Accuracy of Results}

In order to confirm the accuracy of results, power $P$ was calculated by comparing two independent calculation methods. Equation (9)

$$
I \dot{\varphi} \frac{\mathrm{d} \dot{\varphi}}{\mathrm{d} T}=P-C \dot{\varphi}^{2} \Rightarrow P=I \dot{\varphi} \frac{\mathrm{d} \dot{\varphi}}{\mathrm{d} T}+C \dot{\varphi}^{2}
$$

yields one power value $\left(\boldsymbol{P}_{\mathbf{1}}\right)$ and the other one $\left(\boldsymbol{P}_{\mathbf{2}}\right)$ comes from the curve fitting used in Figures 5 and $7(P / C)$.

In forearm rotation downwards the angular acceleration at point $T=0.10 \mathrm{~s}, \dot{\varphi}=14.5 \mathrm{rad} / \mathrm{s}$ was calculated by using the tangent of the angular velocity curve (Figure 8). The tangent point can be found because the tangent has only one point on the curve, otherwise there are two intersection points. The value of angular acceleration in Figure 8 was calculated according to

$$
\frac{\mathrm{d} \dot{\varphi}}{\mathrm{d} T}=14.5 / 0.121 / \mathrm{s}^{2}=1211 / \mathrm{s}^{2} .
$$

This value of angular velocity derivative can also be calculated using Equation (15). The time and angular velocity of this equation corresponding to the measured angular velocity curve time $0.10 \mathrm{~s}$ and velocity $14.5 \mathrm{rad} / \mathrm{s}$ was calculated with Equation (13). Substitution of velocity $14.5 \mathrm{rad} / \mathrm{s}$ into Equation (13) gives time $0.031 \mathrm{~s}$. The derivative of Equation (15)

$$
\begin{gathered}
\dot{\varphi}=\sqrt{\frac{P}{C}\left(1-e^{-\frac{2 C}{I} T}\right)}, \\
X_{1}=1-e^{\frac{-2 C}{I} T}, X_{2}=\frac{-2 C}{I} T \\
\frac{\mathrm{d} \dot{\varphi}}{\mathrm{d} T}=\frac{\mathrm{d} \dot{\varphi}}{\mathrm{d} X_{1}} \cdot \frac{\mathrm{d} X_{1}}{\mathrm{~d} X_{2}} \cdot \frac{\mathrm{d} X_{2}}{\mathrm{~d} T}=\frac{\sqrt{\frac{P C}{I^{2}}} e^{\frac{-2 C}{I} T}}{\sqrt{1-e^{\frac{-2 C}{I}}}}
\end{gathered}
$$

Substituting in this equation $T=0.031 \mathrm{~s}, I=0.11$ $\mathrm{kg} \cdot \mathrm{m}^{2}, C=2.38 \mathrm{~kg} \cdot \mathrm{m}^{2} / \mathrm{s}$ and $P=693 \mathrm{~W}$, the value of angular acceleration of $1121 / \mathrm{s}^{2}$ was obtained. Moment arm of gravitational force is so short at forearm rotation that the power generation of gravitational force has no significance. In whole arm rotation downwards and whole arm rotation upwards the effect of gravitational force is within power $P$. The accuracy of results is presented in Table 2.

\section{Conclusions}

Hypothesis 1: Movement proceeds at a constant maximal rotational moment. Measurements of the rotation movements show that movement proceeds at a constant angular acceleration between A-B. Therefore, it can be concluded that the torque accelerating the movement or the 
Table 2. Determination of accuracy of the results. The accuracy was obtained as a difference between the power values $P_{1}$ (from Equation (16)) and $P_{2}$ (from the curve fitting in Figures 5 and 7).

\begin{tabular}{|c|c|c|c|}
\hline & Forearm rotation & & \\
\hline & Down (Figure 4) & Down (Figure 6) & Up (Figure 6) \\
\hline Time $(T)$ & $0.100 \mathrm{~s}$ & $0.160 \mathrm{~s}$ & $0.114 \mathrm{~s}$ \\
\hline Angular velocity $(\dot{\varphi})$ & $14.5 \mathrm{rad} / \mathrm{s}$ & $13.3 \mathrm{rad} / \mathrm{s}$ & $11 \mathrm{rad} / \mathrm{s}$ \\
\hline Angular acceleration $\left(\frac{\mathrm{d} \dot{\varphi}}{\mathrm{d} T}\right)$ & $14.5 / 0.121 / \mathrm{s}^{2}$ & $13 / 0.161 / \mathrm{s}^{2}$ & $11 / 0.171 / \mathrm{s}^{2}$ \\
\hline Moment of inertia $(I)$ & $0.11 \mathrm{~kg} \cdot \mathrm{m}^{2}$ & $0.52 \mathrm{~kg} \cdot \mathrm{m}^{2}$ & $0.52 \mathrm{~kg} \cdot \mathrm{m}^{2}$ \\
\hline Power into acceleration $\left(I \dot{\varphi} \frac{\mathrm{d} \dot{\varphi}}{\mathrm{d} T}\right)$ & $193 \mathrm{~W}$ & $562 \mathrm{~W}$ & $370 \mathrm{~W}$ \\
\hline Coefficient of friction $(C)$ & $2.38 \mathrm{~kg} \cdot \mathrm{m}^{2} / \mathrm{s}$ & $3.0 \mathrm{~kg} \cdot \mathrm{m}^{2} / \mathrm{s}$ & $3.0 \mathrm{~kg} \cdot \mathrm{m}^{2} / \mathrm{s}$ \\
\hline Power into friction $\left(C \dot{\varphi}^{2}\right)$ & $500 \mathrm{~W}$ & $531 \mathrm{~W}$ & $363 \mathrm{~W}$ \\
\hline Muscle Power $\left(P_{1}\right)$ & $693 \mathrm{~W}$ & $1093 \mathrm{~W}$ & $733 \mathrm{~W}$ \\
\hline Power/Coefficient of friction $(P / C)$ & $2851 / \mathrm{s}^{2}$ & $3601 / \mathrm{s}^{2}$ & $2501 / \mathrm{s}^{2}$ \\
\hline Muscle Power $\left(P_{2}\right)$ & $678 \mathrm{~W}$ & $1080 \mathrm{~W}$ & $750 \mathrm{~W}$ \\
\hline Error $\frac{P_{1}-P_{2}}{0.5\left(P_{1}+P_{2}\right)} 100$ & $2.2 \%$ & $1.2 \%$ & $2.3 \%$ \\
\hline
\end{tabular}

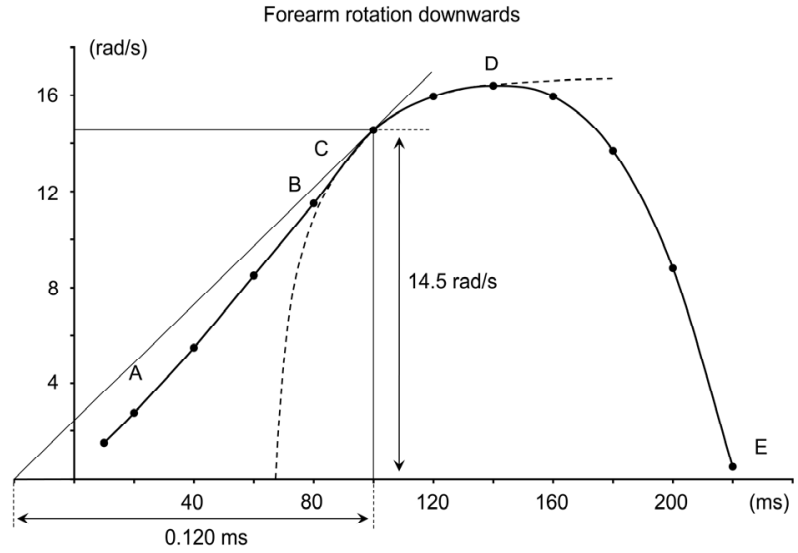

Figure 8. Calculation of angular acceleration at point $(T=$ $0.10 \mathrm{~s}, \dot{\varphi}=14.5 \mathrm{rad} / \mathrm{s})$, where the theoretical angular velocity curve (broken line) coincides with the measured angular velocity curve (points) between $\mathrm{C}$-D.

left side of Equation (8) is constant.

$$
I \frac{\mathrm{d} \dot{\varphi}}{\mathrm{d} T}=\frac{P}{\dot{\varphi}}-C \dot{\varphi}
$$

Torque accelerating the movement is not the same as muscle force which is included in the term $P / \dot{\varphi}$. Therefore, we can conclude that Hypothesis 1 is not fulfilled. However, "movement proceeds at a constant acceleration" is an interesting finding which should be studied more closely. In Equation (8) kinetic friction was assumed to be directly proportional to velocity between A-B. This is a third hypothesis included into this study, which is not necessarily true. It is possible that kinetic friction is constant at small velocities and at large velocities directly proportional to velocity. Then there is a constant torque value accelerating the movement between A-B. The constant acceleration of the velocity curve may be related to the evolution of the human beings. For example the smooth acceleration may be essential for the accuracy of javelin throwing and targeting in fighting and hunting. As mentioned in [10] when modeling the control of the human limb motions, the final aim is to estimate the force production of individual muscles involved. Therefore the constant acceleration theory may play important role in human movements.

Hypothesis 2: movement proceeds at a constant maximal muscle power. Since the matched range (C-D) of the theoretical and measured velocity curves of arm rotation was long enough, it can be clearly seen that the curves did not intersect each other. Therefore it can be inferred that the constant maximum power hypothesis is true between C-D. In addition to the present study of three different type of arm rotation experiments the model of constant maximum power was also fulfilled in the previous experiments of shot put [11]. The different arm movements used in these experiments helped to achieve a greater certainty for the functioning ability of the present model. This model can be considered the most interesting finding of the present study.

\section{REFERENCES}

[1] A. V. Hill, "The Heat of Shortening and the Dynamic Constants of Muscle," Proceedings of the Royal Society of London, Vol. 126, No. 843, 1938, pp. 136-195. 
[2] A. V. Hill, "First and Last Experiments in Muscle Mechanics," Cambridge University Press, Cambridge, 1970.

[3] W. Herzog, "Force-Velocity Relation," In: B. M. Nigg and W. Herzog, Eds., Biomechanics of the MusculoSkeletal System, 2nd Edition, John Wiley \& Sons Ltd, Chichester, 1999, pp. 173-180.

[4] W. Herzog, "Mechanical Properties and Performance in Skeletal Muscles," In: V. Zatsiorsky, Ed., Biomechanics in sport, Blackwell Science University Press, Cambridge, 2000, pp. 21-32. doi:10.1002/9780470693797.ch2

[5] B. R. MacIntosh and R. J. Holash, "Power Output and Force Velocity Properties of Muscle," In: B. M. Nigg, B. R. MacIntosh and J. Mester, Eds., Biomechanics and Biology of Movement, Human Kinetics, Champaign, 2000, pp. 193-210.

[6] D. A. Winter, "Biomechanics and Motor Control of $\mathrm{Hu}-$ man Movement," 3rd Edition, John Wiley \& Sons Inc., Hoboken, 2004, pp. 215-222.

[7] J. H. Challis, "Muscle-Tendon Architecture and Athletic performance," In: V. Zatsiorsky, Ed., Biomechanics in sport, Blackwell Science University Press, Cambridge, 2000, pp. 33-55. doi:10.1002/9780470693797.ch3

[8] D. E. Rassier, B. R. MacIntosh and W. Herzog, "Length Dependence of Active Force Production in Skeletal Muscle," Journal of Applied Physiology, Vol. 86, No. 5, 1999 , pp. $1445-1457$.

[9] R. T. Raikova, "A Model of the Flexion - Extension Motion in the Elbow Joint-Some Problems Concerning Muscle Forces Modeling and Computation," Journal of Biomechanics, Vol. 29, No. 6, 1996, pp. 763-772. doi:10.1016/0021-9290(95)00072-0

[10] R. T. Raikova and H. Ts. Aladjov, "Comparison between Two Models under Dynamic Conditions," Computers in Biology and Medicine, Vol. 35, No. 5, 2005, pp. 373-387. doi:10.1016/S0010-4825(04)00041-1

[11] A. Rahikainen and P. Luhtanen, "A Study of the Effect of Body Rotation on the Arm Push in Shot Put," Russian Journal of Biomechanics, Vol. 8, No. 2, 2004, pp. 78-93.

[12] A. Rahikainen, "Biomechanics in Shot Put," Helsinki University, Helsinki, 2008.

[13] A. Rahikainen, J. Avela and M. Virmavirta, "Modeling the Force-Velocity Relationship in Arm Movement," Proceedings of the 14th ECSS Congress, Oslo, 24-27 June 2009, p. 570.

[14] A. Rahikainen, "Method and Apparatus for Photographing a Movement," US Patent No. 4927261, 1990.

[15] A. Rahikainen, "The Use of Rotating Disk in the Photography of Movements," Russian Journal of Biomechanics, Vol. 7, No. 1, 2003, pp. 47-64. 